\title{
Dynamic F-Wave Study Aids in Diagnosis of Neurogenic Thoracic Outlet Syndrome
}

\author{
Jin Jun Luo ${ }^{1,2}$ and Favio Bumanlag ${ }^{1}$ \\ ${ }^{1}$ Department of Neurology \\ ${ }^{2}$ Department of Pharmacology
}

Received: November 30, 2013; Accepted: November 30, 2013; Published: November 30, 2013

*Corresponding author: Dr. J.J. Luo, Department of Neurology, 3401 N. Broad Street, C525, Philadelphia, PA 19140, USA. Tel: 1-215-707-3915. E-mail: luo@temple.edu

\begin{abstract}
Neurogenic thoracic outlet syndrome (TOS) is a clinical symptom complex that results from recurrent mechanical compression of the brachial plexus in the region between the base of the neck and the armpit. Its diagnosis may, at times, be elusive due to the lack of sensitivity or inconsistency in the laboratory evaluations. Upper extremity neurodiagnostic studies, such as nerve conduction studies, electromyography, and somatosensory evoked potentials, are of limited value in making an early diagnosis of TOS. Herein, we report measurements of the mean latency difference in dynamic F-wave studies helps in the early diagnosis of TOS.
\end{abstract}

\section{Abbreviations}

CT: Computerized Tomography; EMG: electromyography; MLD: mean latency difference; MRI: magnetic resonance imaging; NCS: nerve conduction study; TOS: thoracic outlet syndrome

\section{Introduction}

Neurogenic thoracic outlet syndrome (TOS) is a type of brachial plexopathy caused by recurrent microinjury in the region between the base of the neck and the armpit. Electrophysiologic diagnosis of TOS remains a challenge [1]. A number of electrodiagnostic approaches have been explored including nerve conduction studies (NCS), electromyography (EMG), and C8 nerve root stimulation [2-4]. Abnormal findings of medial antebrachial sensory in NCS and denervation changes in C8-T1 myotomeson EMG may provide electrophysiologic evidence to support the diagnosis $[2,3,5]$. Unfortunately, the frequency of the electrodiagnostic yield is usually low. Use of routine F-wave study and somatosensory evoked potential are of limited value [5-7]. Imaging studies including ultrasound, MRI, or CT angiography may show pathology in severe cases, but in early stages sensitivity of such testing is low [8]. Recently, CuevasTrisan and Cruz-Jimenez reported that provocative $\mathrm{F}$ waves may help in early diagnosis [9]. However, no significant changes in provocative $\mathrm{F}$ waves in the diagnosis of nonspecific neurogenictype thoracic outlet syndrome were lately reported, indicating absence of acceptable sensitivity in this regard [10]. Here we report that measurements of the mean latency difference in dynamic F-wave study may increase the diagnostic sensitivity and aid in the diagnosis of TOS.

\section{Patients and electrophysiologic studies}

Seven subjects $[37.7 \pm 17.3$ year-old, mean $\pm S D$, range 18 to $62 \mathrm{y}$, female/Male $=6 / 1]$ with a clinically provisional diagnosis of TOS were studied [Table 1]. All had chronic symptoms [27.3 \pm 31.1 months, range from 5 to 96] including paresthesias or weakness involving one upper extremity. All had at least one positive on provocative [Adson, Wright and costoclavicular] maneuvers but negative on Spurling's. Neuroimaging studies excluded the presence of a cervical rib or radiculopathy. NCS was performed on median and ulnar motor and sensory nerves; and on radial, medial and lateral antebrachial sensory nerves. Needle EMG was performed on muscles including deltoid, biceps, triceps, first dorsal interposes, abductor pollicis brevis, and flexor carpi ulnaris.

Dynamic F-wave study was performed on patients lying supine with the arms placed in two positions: 1) The relaxed position in which the arms were placed at the side, parallel to the length of the torso; and 2) The stressed position in which the arms were flexed and raised so that the humerus was parallel to the length of the head. The minimal, mean, and maximal latencies, chrono dispersion and amplitudes were recorded. Simultaneously, real-time changes of skin temperature were monitored and recorded. Within two minutes of the arm placed in the stressed position all subjects complained of paresthesias, and/or "swollen" feeling involving the fingers, hand, or forearm which resolved within 30 seconds after returning the arm to the relaxed position. The data were obtained by averaging the mean latencies of the F-waves from a pool of $2-3$ trials, in which each consisted of 15 recordings provoked by consecutive super-maximal stimulation. The mean latency difference [MLD] following the dynamic study was obtained by subtraction of the latency recorded at the relaxed position from those recorded at the stressed position $\left[\mathrm{MLD}=\right.$ Latency $_{[\text {Stressed] }}$-Latency $\left.{ }_{[\text {Relaxed] }}\right]$.

\section{Results and Discussion}

In all but 2 subjects who possessed carpal tunnel syndrome, 
Table 1: Demographic data and electrodiagnostic findings.

\begin{tabular}{|c|c|c|c|c|c|c|c|c|c|c|c|c|c|c|c|c|}
\hline Age/Sex & $\begin{array}{l}\text { Symp- } \\
\text { toms }\end{array}$ & Duration & NCS & EMG & $\begin{array}{c}\text { L- } \\
\text { UlanrRlx }\end{array}$ & $\begin{array}{c}\text { L- } \\
\text { UlanrStr }\end{array}$ & $\begin{array}{c}\text { R- } \\
\text { UlanrRIx }\end{array}$ & $\begin{array}{c}\text { R- } \\
\text { UlanrStr }\end{array}$ & $\begin{array}{l}\text { L-Medi- } \\
\text { anRlx }\end{array}$ & $\begin{array}{l}\text { L-Medi- } \\
\text { anStr }\end{array}$ & $\begin{array}{c}\text { R-Me- } \\
\text { dian } \\
\text { Rlx }\end{array}$ & $\begin{array}{c}\text { R-Me- } \\
\text { dian } \\
\text { Str }\end{array}$ & $\begin{array}{c}\text { L-Ul- } \\
\text { nar } \\
\text { MLD }\end{array}$ & $\begin{array}{l}\text { R-Ulnar } \\
\text { MLD }\end{array}$ & $\begin{array}{c}\text { L-Me- } \\
\text { dian } \\
\text { MLD }\end{array}$ & $\begin{array}{c}\text { R-Medi- } \\
\text { an } \\
\text { MLD }\end{array}$ \\
\hline $25 / F$ & Right & 5 & NL & C8nc & 23.78 & 23.54 & 23.16 & 23.88 & 22.33 & 23.5 & 22.63 & 25.09 & -0.23 & 0.73 & 1.17 & 2.45 \\
\hline $30 / \mathrm{F}$ & Right & 12 & CTS & NL & 22.59 & 23.18 & 23.95 & 24.22 & ND & ND & 25.59 & 26.2 & 0.58 & 0.27 & ND & 0.62 \\
\hline $50 / \mathrm{F}$ & Left & 18 & $\begin{array}{l}\mathrm{b} / \mathrm{l} \\
\mathrm{CTS}\end{array}$ & $\begin{array}{l}\text { L C6- } \\
\text { T1nc, } \\
\text { R C7- } \\
\text { 8nc }\end{array}$ & 32.93 & 33.75 & 30.97 & 30.1 & 31.65 & 31.13 & 32.54 & 32.94 & 0.82 & -0.87 & -0.52 & 0.4 \\
\hline $62 / F$ & Left & 24 & NL & $\begin{array}{l}\text { C8- } \\
\text { T1nc }\end{array}$ & 26.51 & 25.98 & 26.96 & 27.53 & 26.64 & 27.99 & 25.33 & 26.52 & -0.53 & 0.57 & 1.35 & 1.18 \\
\hline $18 / \mathrm{M}$ & Left & 12 & NL & C8nc & 27.29 & 27.03 & 27.97 & 28.71 & 25.4 & 27.8 & 27.05 & 27.16 & -0.26 & 0.74 & 2.4 & 0.1 \\
\hline $54 / \mathrm{F}$ & Left & 24 & NL & NL & 27.08 & 29.02 & 26.65 & 27.08 & 27.52 & 27.41 & ND & ND & 1.94 & 0.43 & -0.11 & ND \\
\hline $27 / F$ & Left & 96 & NL & $\begin{array}{l}\text { C8- } \\
\text { T1nc }\end{array}$ & 25.27 & 26.03 & 25.09 & 25.21 & 23.94 & 23.84 & 23.66 & 23.54 & 0.76 & 0.12 & -0.1 & -0.12 \\
\hline
\end{tabular}

Cutoffs (CI: 99\%) $1.89 \mathrm{~ms} 2.09 \mathrm{~ms}$

*Mean \pm SD

F: female; M: male; ys: years; mos: months; ms: milliseconds; NCS: nerve conduction study; EMG: electromyography.

L: left; R: right; NL: normal; Rlx: relaxed/neutral position of the arm; Str: stressed/hyperextension of the arm;

MLD: mean latency difference $\left\{\right.$ MLD=Latency ${ }_{[\text {Stressed] }}-$ Latency $\left._{[\text {Relaxed }}\right\}$; b/l: bilateral;

CTS: carpal tunnel syndrome; nc: neurogenic changes; C7, C7, C8,T1: myotomes; ND: not done;

CI: confidence level.

a normal motor and sensory NCS was obtained [Table 1]. EMG demonstrated mild neurogenic changes such as polyphasia in the muscles of first dorsal interosseus, abductor pollicis brevis, and flexor carpi ulnaris, which were innervated by the lower trunk of the cervical plexus, in 5 subjects (Table 1). A significantly prolonged MLD was observed in the 3 subjects who were symptomatic (Table 1), suggesting position-related conduction slowing. The calculated mean values of MLD were $0.24 \pm 0.55$ $\mathrm{ms}$ in the ulnar [ $\mathrm{n}=14]$ and $0.29 \pm 0.60 \mathrm{~ms}$ in the median $[\mathrm{n}=12]$ nerves. The calculated normal cutoffs of MLD [at the 3 standard deviation, 99\% confidence level] in our series are $1.89 \mathrm{~ms}$ for the ulnar and $2.09 \mathrm{~ms}$ median nerve, respectively. Variation of the changes in minimal and maximal latencies, chronodispersion, and amplitudes of the F-waves was observed but was without statistical significance [data not shown]. No statistically significant skin temperature changes were noted [data not shown].

Dynamic F-wave study is a non-invasive, well tolerated technique which is relatively easy to perform. It may provide additional diagnostic value in the early stages of TOS. Our results confirmed the previous notion by Cuevas-Tristan and CruzJimenez that provocative maneuvers may help to uncover the subtle electrodiagnostic abnormalities related to TOS [9] though the sensitivity is low when compared the F-wave latency between the affected and the unaffected arms [10]. Measurements of MLD may increase the sensitivity and permit early diagnosis, especially when normal NCS are encountered. Upper extremity MLD may, however, have limited utility in the setting of a concomitant neuropathy, C8/T1 radiculopathy or polyradiculoneuropathy.

In summary, our findings suggest that analyzing MLD from the dynamic F-wave measurement may be a useful addition to conventional electrophysiological study in early diagnosis of TOS.
A study in a large number of patients with TOS may be needed to validate the findings. Additionally, normative MLD values may be established individually from individual electrodiagnostic laboratories.

\section{Acknowledgments}

The authors are grateful to John Kincaid, MD, of Neurology at Indiana University School of Medicine for his valuable comments on this manuscript.

\section{References}

1. Rousseff R, Tzvetanov P, Valkov I (2005) Utility (or futility?) of electrodiagnosis in thoracic outlet syndrome. Electromyogr Clin Neurophysiol 45(3): 131-133.

2. Cruz-Martinez A, Arpa J (2001) Electrophysiological assessment in neurogenic thoracic outlet syndrome. ElectromyogrClinNeurophysiol 41(4): 253-256.

3. Nishida T, Price SJ, Minieka MM (1993) Medial antebrachial cutaneous nerve conduction in true neurogenic thoracic outlet syndrome. ElectromyogrClinNeurophysiol;33(5): 285-288.

4. Pavot AP, Ignacio DR, Gargour GW (1989) Assessment of conduction from $c 8$ nerve root exit to supraclavicular fossa--its value in the diagnosis of thoracic outlet syndrome. ElectromyogrClinNeurophysiol 29(7-8): 445451.

5. Aminoff MJ, Olney RK, Parry GJ, Raskin NH(1988) Relative utility of different electrophysiologic techniques in the evaluation of brachial plexopathies. Neurology 38(4):546-550.

6. Veilleux M, Stevens JC, Campbell JK (1988) Somatosensory evoked potentials: Lack of value for diagnosis of thoracic outlet syndrome. Muscle Nerve 11(6):571-575.

7. Passero S, Paradiso C, Giannini F, Cioni R, Burgalassi L et al. (1994) 
Diagnosis of thoracic outlet syndrome. Relative value of electrophysiological studies. Acta Neurol Scand 90(3):179-185.

8. Demondion X, Herbinet P, Van Sint Jan S, Boutry N, Chantelot C, Cotten A (2006) A Imaging assessment of thoracic outlet syndrome. Radiographics 26(6):1735-1750.
9. Cuevas-Trisan RL, Cruz-Jimenez M (2003) Provocative f waves may help in the diagnosis of thoracic outlet syndrome: A report of three cases. Am J Phys Med Rehabil 82(9):712-715.

10. Ozgonenel L, Akyuz G, Ozgonenel B, Adatepe T (2012) Provocative f wave in the diagnosis of nonspecific neurogenic-type thoracic outlet syndrome. Am J Phys Med Rehabil 91(4):316-320. 